\title{
Emission and Focusing Characteristics of a Quintuple-gated Field Emitter Array
}

Akifumi Koike ${ }^{1}$, Tomoya Tagami ${ }^{1}$, Yasuo Takagi ${ }^{1}$, Masayoshi Nagao ${ }^{2}$, Tomoya Yoshida ${ }^{2}$, Toru Aoki $^{1}$, Yoichiro $\mathrm{Neo}^{1}$ and Hidenori Mimura ${ }^{1}$

${ }^{1}$ Research Institute of Electronics, Shizuoka University, Hamamatsu, Japan, 432-8011

${ }^{2}$ National Institute of Advanced Industrial Science and Technology (AIST), Tsukuba, Ibaraki, Japan, 305-8568

A field emitter array (FEA) with a five-stacked gate electrode, that is, a quintuple-gated FEA with a four-stacked electrode lens, was fabricated. The four-stacked electrode forms an einzel lens where the second and third electrodes are connected through a contact hole. Because the electron velocity of the einzel lens in a quintuple-gated FEA is smaller than that of the quadruple-gated FEA, the quintuple-gated FEA has a stronger electron convergence. The slit sweeping method confirmed that a beam crossover is formed for the quintuple-gated FEA.

A field emitter array (FEA) with a focusing electrode is an appealing device for electron beam lithography, ${ }^{1)}$ high definition field emission displays, ${ }^{2)}$ and image sensors. ${ }^{3)}$ Previously, the fabrication and characteristics of a double-gated FEA with two-stacked electrodes have been reported. ${ }^{4-6)}$ Double-gated FEAs can effectively generate a focused electron beams. However, one drawback to a double-gated FEA is drastic reduction in the emission current under the focusing condition due to the decreased field enhancement at the emitter tip caused by the low potential of the vicinal focusing electrode. ${ }^{4)}$ Recently, we reported a volcano structured field emitter comprised of a focusing electrode located below the extraction gate (Gex) electrode. ${ }^{7,8)}$ This volcano structured FEA is the first to focus the electron beam without significantly decreasing the emission current. However, some electrons still cannot penetrate the potential barrier formed by the focusing electrode potential under a strong focusing condition, and these electrons travel back to the Gex electrode. To overcome these issues, we have developed a quadruple-gated (quad-gated) FEA with a three-stacked electrode electrostatic lens. ${ }^{9)}$ The first electrode (G1), which is near the Gex electrode, maintains the emitter tip, and hence, requires a voltage greater than the Gex voltage. The second electrode (G2) focuses the electron beam, and requires a voltage less than the Gex voltage. The third electrode (G3) inhibits the formation of a potential barrier on the electron trajectory, and therefore, requires a voltage greater than the G2 voltage. We applied the same voltage to G1 and G3, and the three-stacked electrode consequently formed an einzel lens. Typically we applied 50, 100, and $100 \mathrm{~V}$ to Gex, G1 and G3, respectively, and changed the G2 voltage from 100 (nonfocusing 
condition) to $-30 \mathrm{~V}$ (focusing condition). The electron beam diameter decreased as the G2 voltage decreased, demonstrating that the electron beam was focused. Although the emission current decreased gradually with the G2 voltage, the observed degradation was insignificant. A G2 voltage less than -30 V should show improved focusing characteristics such as the formation of an electron beam crossover. However, the device broke when the voltage applied on G2 was less than $-30 \mathrm{~V}$. Therefore, the electron beam crossover is not formed in the quad-gated FEA. If a beam crossover is formed for the FEA with a built-in einzel lens, then an extremely tiny scanning electron microscope (SEM) using FEA can be realized. To form a crossover, a more effective converging field must be formed. Therefore, in the present study, we added one more gate electrode to the three-stacked electrode lens. The four-stacked electrode lens forms in the region where the electron velocity becomes smaller than that of the three-stacked electrode lens, and because the qui-potential lines penetrate toward the smaller electron velocity region, a more effective converging field is formed. ${ }^{10)}$ Herein we describe the emission and focusing characteristics of a quintuple-gated FEA with a four-stacked electrode lens. In our previous papers, ${ }^{7,9)}$ the beam size was evaluated by the size of the bright spot excited by the electron beam on a phosphor screen. However, the beam size could not be precisely estimated because the bright area excited by the electron beam on a phosphor is blurred. Although the knife-edge sweeping method is useful to measure the ion beam width, ${ }^{11}$ ) the knife-edge is a one-sided plate that easily attracts or repels the electron beam and consequently, changes the electron beam trajectory. Therefore, we have developed a slit sweeping method to measure the electron beam width. The slit sweeping method clearly shows that an electron beam crossover was formed for a quintuple-gated field emitter.

Figure 1 depicts a cross-sectional SEM image of the quintuple-gated field emitter. The fabrication process of the quintuple-gated FEA is similar to that of the quad-gated FEA. ${ }^{9}$ ) To sufficiently decrease the electron velocity, G2 and G3 were connected through a contact hole; hereafter we describe the second and third gates $\mathrm{G} 2,3$. The $\mathrm{SiO}_{2}$ insulating layer between the Si tip and Gex was $250 \mathrm{~nm}$ thick, while the other intermediate insulating layers were 350, 750, 750 and $350 \mathrm{~nm}$ thick from the bottom side. Although the intermediate insulating layer was $500 \mathrm{~nm}$ in the quad-gated FEA, the thickness of the intermediate layer was increased to further separate G1 and G3. Each gate electrode was composed of $100 \mathrm{~nm}$ thick niobium. The electron emission and focusing characteristics were evaluated in an ultrahigh-vacuum chamber at a pressure of $1 \times 10^{7} \mathrm{~Pa}$.

Figure 2 shows the measurement system using a phosphor screen. The $1 \mathrm{kV}$ anode phosphor screen bias was located $1 \mathrm{~mm}$ above the FEA substrate. The focusing characteristics, which were the anode phosphor current and the diameter of the bright area excited by the electron beam on the anode phosphor as functions of the G2,3 voltage, were measured under the following conditions. The voltages of Gex, G1, and G4 were fixed at 50, 100, and $100 \mathrm{~V}$, 


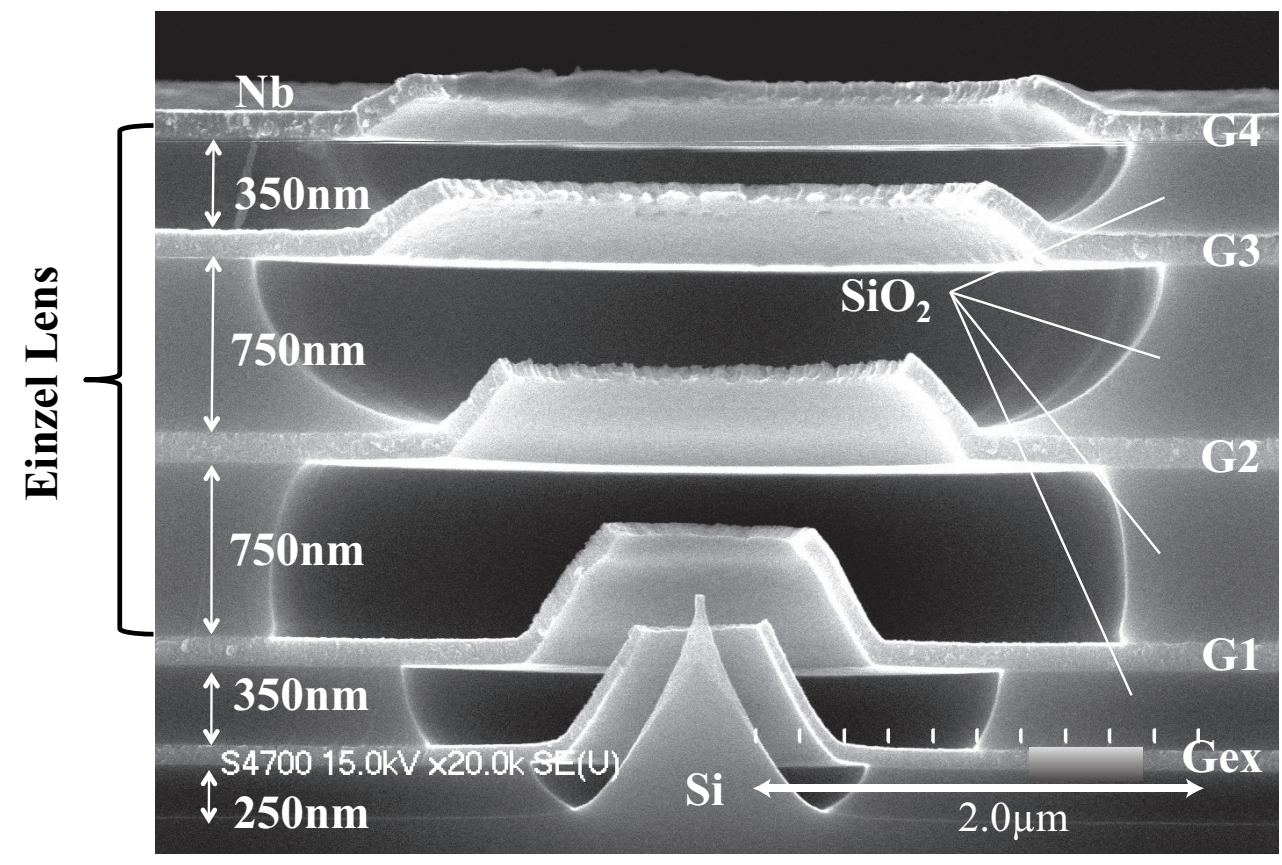

Fig. 1. Cross-sectional SEM image of a quintuple-gated field emitter.

\section{Phosphor Screen}

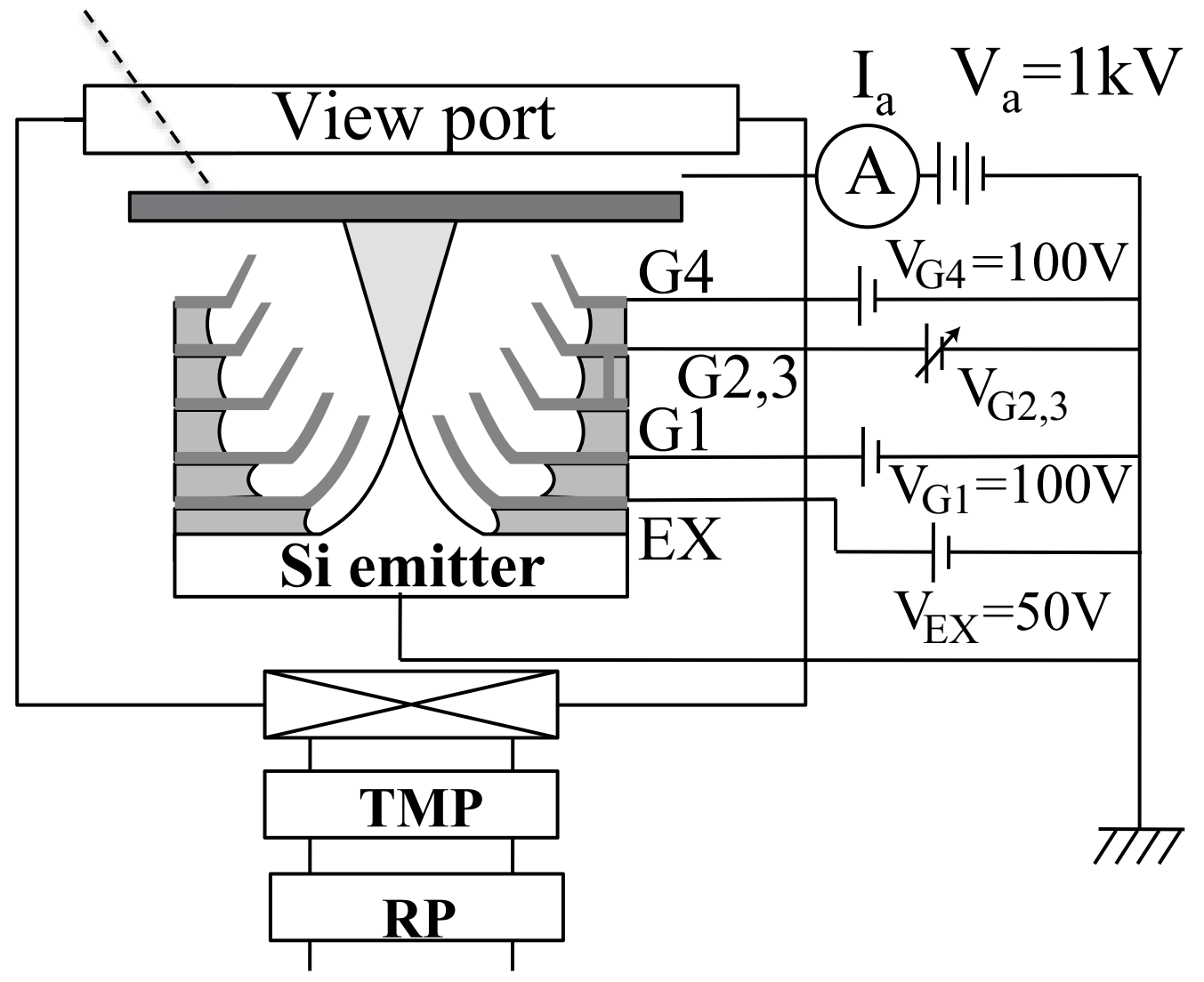

Fig. 2. Measurement system using a phosphor screen. 
respectively. The G2,3 voltage was changed from 100 (nonfocusing condition) to $-10 \mathrm{~V}$ (focusing condition). Voltages for Gex and G1 were chosen to ensure a sufficient anode current of several $\mu \mathrm{A}$ while observing the spot on the phosphor.

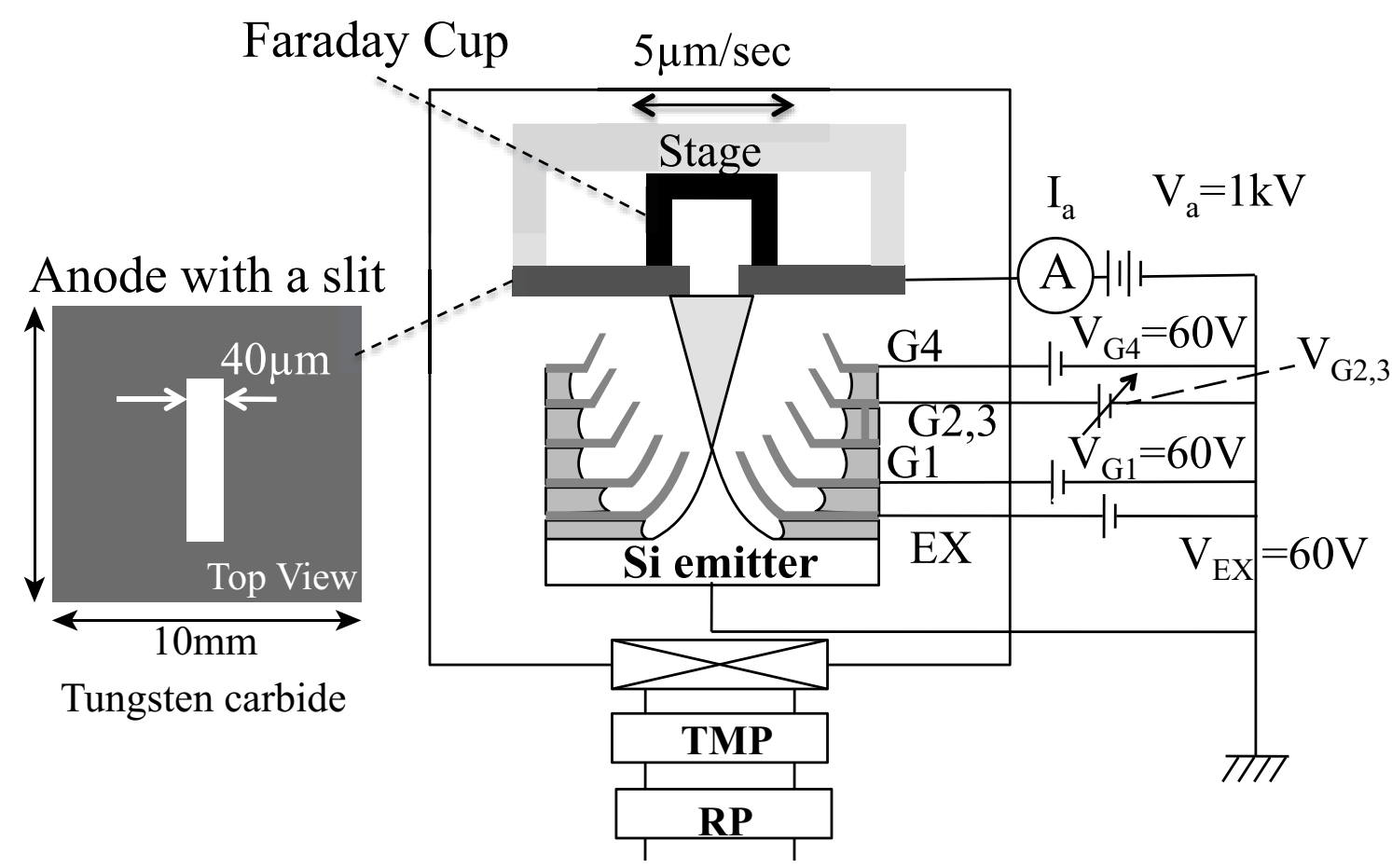

Fig. 3. Schematic of the slit sweeping method to measure the electron beam width.

Figure 3 schematically depicts the slit weeping method that we developed to measure the electron beam width. A tungsten carbide anode with a slit, which measured $40 \mu \mathrm{m}$ wide and $10 \mathrm{~mm}$ long, was placed $1 \mathrm{~mm}$ above the quintuple-gated FEA. A Faraday cup was placed behind the slit and the electron beam passing through the slit was collected by the Faraday cup. The slit and Faraday cup were moved in one direction by a stepping motor at a speed of $5 \mu \mathrm{m} / \mathrm{s}$. The beam width was evaluated using the beam current measured by the Faraday cup downstream of the slit moving across the beam pass. To ensure that the anode current is similar to the current used in an electron microscope (1nA), the voltages of Gex, G1, and G4 were fixed at 60,60 and $60 \mathrm{~V}$, respectively, while the G2,3 voltage was changed from 60 to $0 \mathrm{~V}$. The voltages of the slit plate and Faraday cup were $1 \mathrm{kV}$ and $100 \mathrm{~V}$, respectively. The devices used in Figs. 2 and 3 were single tip field emitters.

Figure 4 shows the anode and gate currents characteristics of the quintuple-gated FEA with seven emitter tips. The potentials of the three-lens electrode were equal to that of the Gex electrode, as schematically shown in the inset. The anode current started at a Gex voltage of approximately $30 \mathrm{~V}$, and an anode current of around $100 \mathrm{nA}$ was obtained at a Gex voltage of 


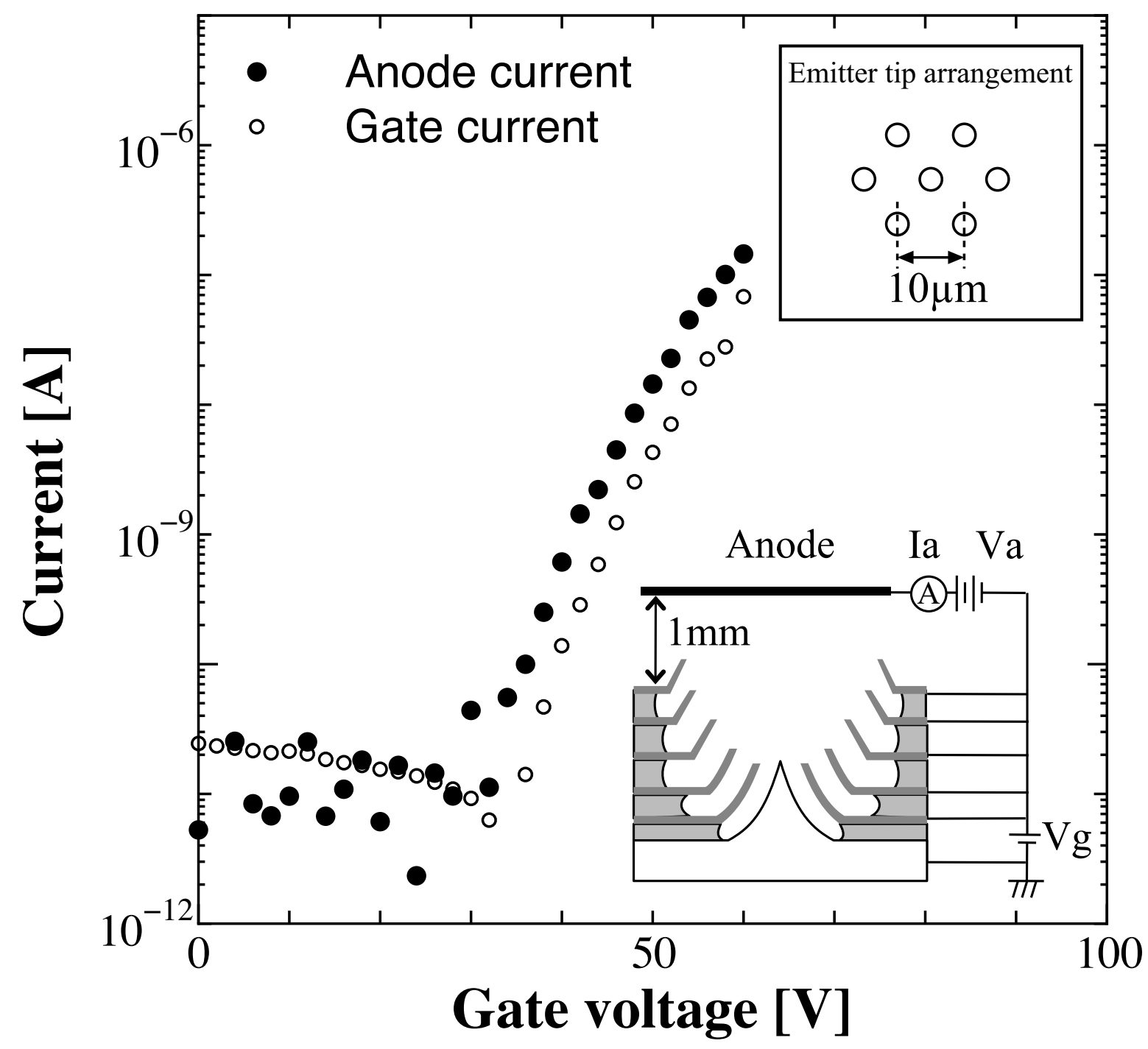

Fig. 4. Anode and gate currents - extraction gate voltage characteristics for the quintuple-gated FEA with seven emitter tips.

$60 \mathrm{~V}$. Therefore, the average emission current of each emitter tip was around $14 \mathrm{nA}$. Compared to the total current entering the all gate electrodes for the quad-gated FEA, the gate current of the quintuple-gated FEA was $10 \%$ higher than that of the quad-gated FEA because more electrons enter the gate electrodes in the nonfocusing condition for the quintuple-gated FEA, which has a larger lens size than the quad-gated FEA.

Figure 5 depicts the beam spots measured from phosphor screen images for the quintuplegated field emitter as well as the phosphor images at G2,3 =100, 10, and -10 V. For comparison, the beam spots for the quad-gated field emitter are also shown. The characteristics were obtained for a single tip quintuple-gated field emitter and single tip quad-gated field emitter. Because both field emitters have different anode currents, the beam sizes cannot be directly 


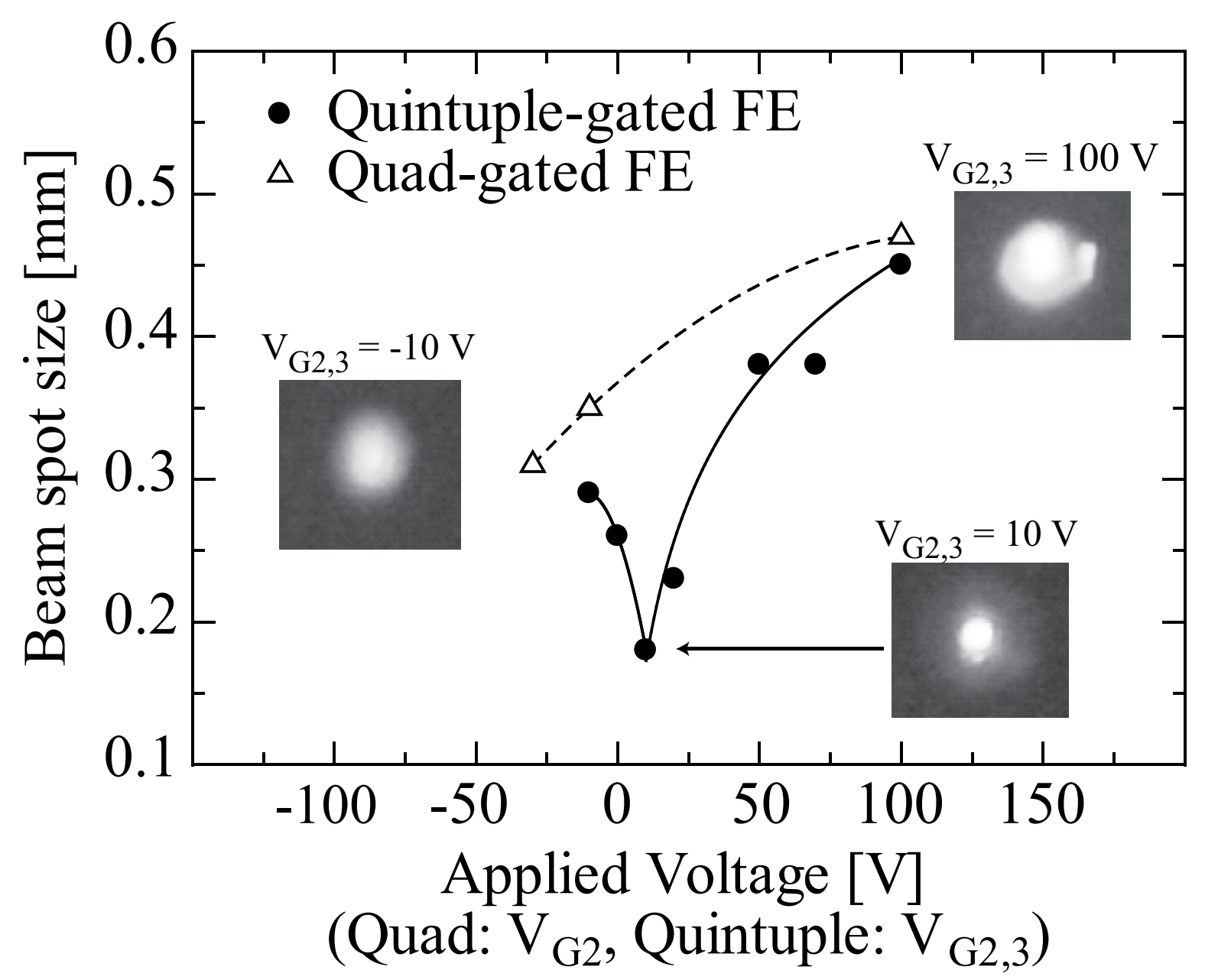

Fig. 5. Beam spots measured from phosphor screen images for a single tip quintuple-gated field emitter (FE) and a single tip quad-gated field emitter. Solid and dashed lines are the least square estimation for the quintuple-gated field emitter and the quad-gated field emitter, respectively.

compared. For the quintuple-gated field emitter, the beam spot decreased as the G2,3 voltage decreased from 100 to $10 \mathrm{~V}$, but then the beam spot increased as the G2,3 voltage went from 10 to $-10 \mathrm{~V}$. On the other hand, the beam spot monotonously decreased as the G2 voltage decreased from 100 to $-30 \mathrm{~V}$ for the quad-gated field emitter. These results suggest that the quintuple-gated field emitter has a more effective converging field and that beam crossover forms for the quintuple-gated field emitter. These observations indicate that forming a region with a smaller electron velocity is useful in creating a more effective converging field.

Figure 6 shows the electron beam widths at three G2,3 voltages $(60,5$, and $0 \mathrm{~V})$ measured by the slit sweeping method. Although the current peak was not clearly observed at a G2,3 voltage of $60 \mathrm{~V}$, the peak was maximized at a G2,3 voltage of $5 \mathrm{~V}$. As the G2,3 voltage decreased from 5 to $0 \mathrm{~V}$, the peak height decreased, but the width increased, indicating a beam crossover is formed between the anode and the field emitter. Because field emitter and 


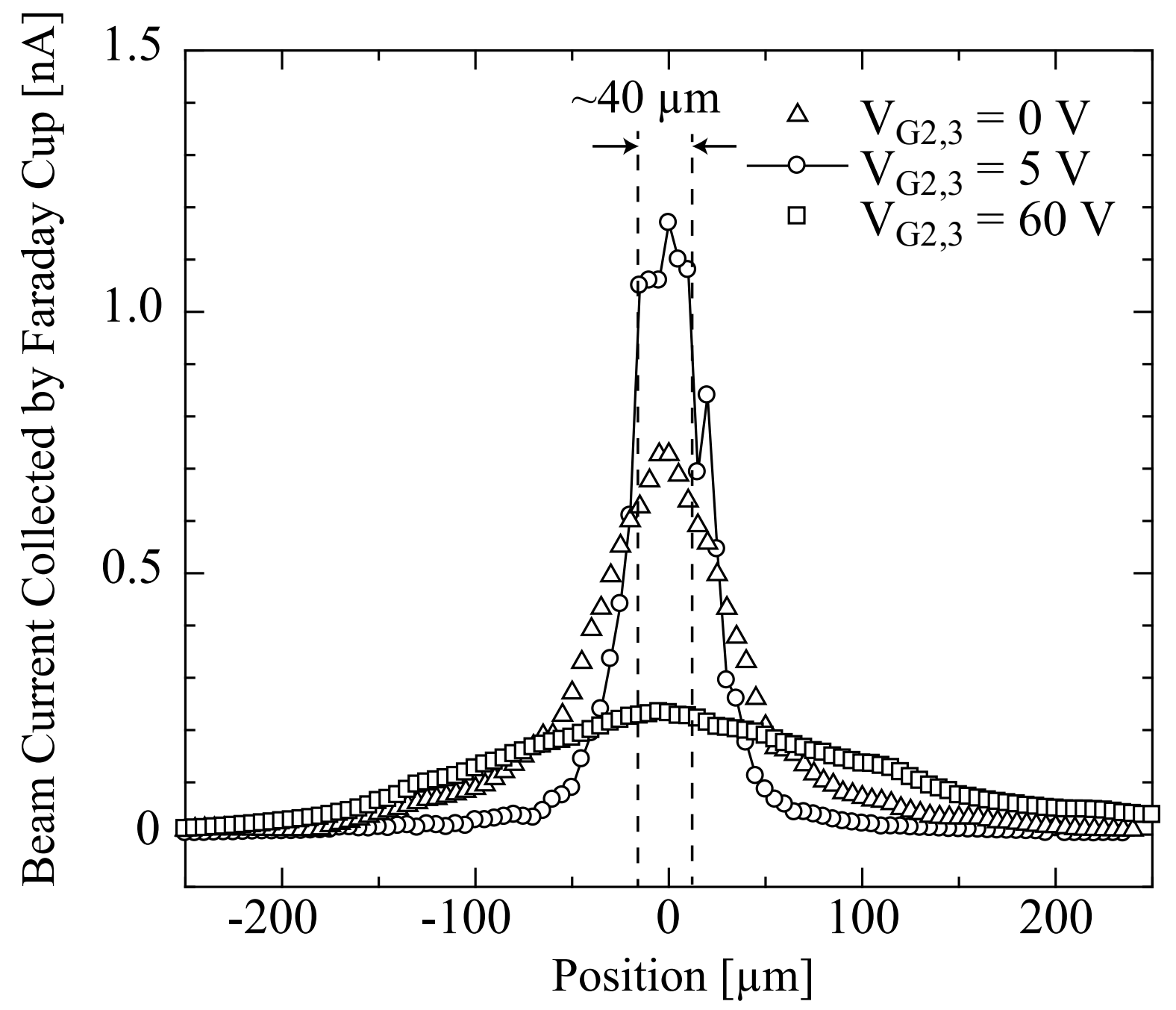

Fig. 6. Electron beam widths at three $\mathrm{G} 2,3$ voltages $(60,5$, and $0 \mathrm{~V})$ measured by the slit sweeping method for a single tip quintuple-gated field emitter. Top width of the peak is around $40 \mu \mathrm{m}$.

slit anode are $1 \mathrm{~mm}$ apart and the crossover is formed immediately in front of the field emitter, this slit sweeping method cannot measure the exact size of the beam crossover. However, the flat shape with about $40 \mu \mathrm{m}$ width at the center of the peak suggests that the width of the beam crossover is less than the slit width of $40 \mu \mathrm{m}$.

In conclusion, we have successfully fabricated a quintuple-gated FEA with a four-stacked electrode lens. Although the quintuple-gated FEA and quad-gated FEA are fabricated using similar processes, the electron convergence for the quintuple-gated field emitter is stronger than that of the quad-gated field emitter. Additionally, the slit sweeping method that we developed confirmed the quintuple-gated field emitter is the first micro-field emitter to form a beam crossover.

Acknowledgements This work was supported by research grants (09A27006a) from the New Energy and Industrial and Technology Development Organization and a Grant-in-Aid for Science 
Research (B) 21310083 from the Japan Society for Promotion of Science (JSPS). 


\section{References}

1) L. R. Bayler, D. H. Lowndes, M. L. Simpson, C. E. Thomas, M. A. Guillorn, V. I. Merkulov, J. H. Whealton, E. D. Ellis, D. K. Hensley, and A. V. Melechko: J. Vac. Sci. technol. B 20 (2002) 2646.

2) K. Hagiwara, T. Takei, T. Sakai, T. Muroi, M. Ushirozawa, and T. Yamamoto: Proc. 12th Int. Display Workshops, 2005, Vol. 2, p.1663.

3) N. Egami, M. Nanba, Y. Takiguchi, K. Miyakawa, T. Watabe, and S. Okazaki: J. Vac. Sci. Technol. B 23 (2005) 2056.

4) Y. Yamaoka, S. Kanemaru, and J. Itoh: Jpn. J. Appl. Phys. 35 (1996) 6626.

5) M. A. Guillorn, A. V. Melechko, V. I. Merkulov, E. D. Ellis, M. L. Simpson, D. H. Lowndes. L. R. Baylor, and G. J. Bordonaro: J. Vac. Sci. Technol. B 19 (2001) 2598.

6) M. A. Guillorn, X. Yang, A. V. Melechko, D. K. Hensley, M. D. Hale, V. I. Merkulov, and M. L. Simpson, L. R. Baylor, W. L. Gardner, and D. H. Lowndes: J.Vac. Sci. Technol. B $22(2004) 35$.

7) Y. Neo, T. Soda, M. Takeda, M. Nagao, T. Yoshida, C. Yasumuro, S. Kanemaru, T. Sakai, K. Hagiwara, N. Saito, T. Aoki, and H. Mimura: Appl. Phys. Express 1 (2008) 053001.

8) T. Soda, M. Nagao, C. Yasumuro, S. Kanemaru, T. Sakai, N. Saito, Y. Neo, T. Aoki, and H. Mimura: Jpn. J. Appl. Phys. 47 (2008) 5252.

9) M. Nagao, T. Yoshida, S. Kanemaru, Y. Neo, and H. Mimura: Jpn. J. Appl. Phys. 48 (2009) 06FK02.

10) P.Grivet: Electron Optics, (Pergamon Press Ltd., Oxford, 1972) 2nd ed., Chap.4, p.85

11) Y. Ishii, A. Isoya, T. Kojima, and K. Arakawa: Nucl. Instrum. Methods Phys. Res. Sect. B 211 (2003) 415. 